\title{
Blood pressure in athletic preparticipation evaluation and the implication for cardiac remodelling
}

\author{
Kristofer Hedman, ${ }^{1,2}$ Kegan J Moneghetti, ${ }^{1,3}$ Jeffrey W Christle, ${ }^{1,3}$ \\ Shadi P Bagherzadeh, 1,2 Myriam Amsallem, 1,2 Euan Ashley, 1,3 Victor Froelicher, 1,3 \\ Francois Haddad ${ }^{1,2}$
}

\begin{abstract}
- Additional material is
published online only. To view, please visit the journal online (http://dx.doi.org/10.1136/ heartjnl-2019-314815).

'Department of Medicine, Division of Cardiovascular Medicine, Stanford, California, USA

${ }^{2}$ Department of Medicine, Stanford Cardiovascular Institute, Stanford, California, USA

${ }^{3}$ Stanford University, Stanford Sports Cardiology, Stanford, California, USA
\end{abstract}

\section{Correspondence to}

Dr Kristofer Hedman, Department of Medicine, Division of Cardiovascular Medicine, Stanford, CA 94025 USA; kristofer.hedman@liu.se

$\mathrm{KH}$ and $\mathrm{KJM}$ contributed equally.

VF and FH contributed equally.

Received 25 January 2019 Revised 26 March 2019 Accepted 4 April 2019 Published Online First 28 May 2019

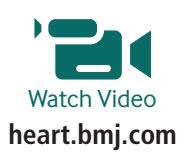

\section{SLinked}

- http://dx.doi.org/10.1136/ heartjnl-2019-315188

\section{Check for updates}

(C) Author(s) (or their employer(s)) 2019. No commercial re-use. See rights and permissions. Published by BMJ.

To cite: Hedman $\mathrm{K}$,

Moneghetti KJ,

Christle JW, et al. Heart

2019:105:1223-1230.

\section{ABSTRACT}

Objectives To explore blood pressure (BP) in athletes at preparticipation evaluation (PPE) in the context of recently updated US and European hypertension guidelines, and to determine the relationship between BP and left ventricular (LV) remodelling.

Methods In this retrospective study, athletes aged 13-35 years who underwent PPE facilitated by the Stanford Sports Cardiology programme were considered. Resting BP was measured in both arms; repeated once if $\geq 140 / 90 \mathrm{~mm} \mathrm{Hg}$. Athletes with abnormal ECGs or known hypertension were excluded. BP was categorised per US/ European hypertension guidelines. In a separate cohort of athletes undergoing routine PPE echocardiography, we explored the relationship between BP and LV remodelling (LV mass, mass/volume ratio, sphericity index) and LV function.

Results In cohort 1 ( $n=2733,65.5 \%$ male), $34.3 \%$ of athletes exceeded US hypertension thresholds. Male sex $(B=3.17, p<0.001)$, body mass index $(B M I)(B=0.80$, $p<0.001)$ and height $(B=0.25, p<0.001)$ were the strongest independent correlates of systolic BP. In the second cohort ( $n=304$, ages 17-26), systolic BP was an independent correlate of $\mathrm{LV}$ mass/volume ratio $(B=0.002$, $\mathrm{p}=0.001)$. LV longitudinal strain was similar across BP categories, while higher BP was associated with slower early diastolic relaxation.

Conclusion In a large contemporary cohort of athletes, one-third presented with BP levels above the current US guidelines' thresholds for hypertension, highlighting that lowering the BP thresholds at PPE warrants careful consideration as well as efforts to standardise measurements. Higher systolic BP was associated with male sex, BMI and height and with LV remodelling and diastolic function, suggesting elevated BP in athletes during PPE may signify a clinically relevant condition.

\section{INTRODUCTION}

Regular physical activity lowers blood pressure (BP) and is an effective strategy for the prevention and treatment of hypertension (HTN). ${ }^{1}{ }^{2}$ However, there are reports of a substantial proportion of athletes fulfilling diagnostic criteria for HTN. $^{3}$ Importantly, as increased BP in youth is prognostic of the development of cardiovascular disease, ${ }^{45}$ screening for HTN in young athletes may have implications for primary prevention. US guidelines have recently revised the thresholds for diagnosing HTN from $\geq 140 / 90$ to $\geq 130 / 80 \mathrm{~mm} \mathrm{Hg}$ in adults ${ }^{6}$ and adolescents. ${ }^{7}$ In contrast, European guidelines currently recommend $\geq 140 / 90 \mathrm{~mm} \mathrm{Hg}$ as the thresholds defining HTN in adults, ${ }^{8}$ as do current guidelines for diagnosing HTN in athletes. ${ }^{9}$ The Stanford Sports Cardiology programme has facilitated preparticipation evaluation (PPE) for precollege, college and professional athletes for the last decade. The lowering of thresholds for HTN at PPE may identify athletes with higher cardiovascular risk, providing an opportunity for primary prevention. This needs to be balanced by the fact that these thresholds used in the new US guidelines may result in higher number of athletes being referred for unnecessary HTN monitoring. ${ }^{10}$ Furthermore, methodology in the assessment of BP at PPE may play a role in the interpretation of these data.

One consequence of chronically elevated BP is left ventricular (LV) remodelling, with increased LV mass (LVM) and concentric remodelling, leading to LV dysfunction. ${ }^{112}$ There is evidence of an impact of BP and HTN on cardiac remodelling and function already in children. ${ }^{13}{ }^{14} \mathrm{LV}$ remodelling is also an established feature of the athlete's heart, ${ }^{15} 16$ however the physiological remodelling is proportional and the LV mass/volume ratio (MVR) and LV function are maintained. ${ }^{17} 18$ Thus, considering the LV remodelling pattern in addition to LVM could provide insight as to the clinical significance of BP assessed during PPE.

The objectives of our study were (i) to evaluate BP measured during PPE in a large heterogeneous cohort of young athletes in the context of recently updated HTN guidelines, (ii) to explore the demographic and anthropomorphic determinants of BP in this population and (iii) to evaluate the impact of $\mathrm{BP}$ on LV remodelling and function in a group of college athletes.

\section{METHODS}

\section{Study design}

This retrospective study was composed of two cohorts (see online supplementary figure 1). All athletes who underwent PPE within the Stanford Clinical PPE Programme between years 2010 and 2016 were considered in cohort 1. This included precollege, college and professional athletes. Inclusion criteria were: (i) age 13-35 years; (ii) complete records for (a) resting BP, (b) type of sport and (c) demographics. Exclusion criteria were (i) initial ECG findings requiring follow-up, 
regardless of final pathology or not; (ii) a previous diagnosis of HTN and/or current BP medication.

Cohort 2 included athletes who underwent routine echocardiography as part of the Stanford PPE Screening Programme between 2008 and 2016. We included athletes with (i) image quality allowing for calculation of LVM and volume; (ii) records of (a) resting BP the same day echocardiography and (b) demographic and anthropomorphic measures. Exclusion criteria were (i) known HTN and/or current BP medication or (ii) pathological findings on echocardiogram. For the purpose of this study, the term 'football' describes American-style gridiron football, and 'soccer' describes the sport in Europe termed football. All participants signed a consent form approved by the institutional review board at Stanford University.

\section{Preparticipation clinical evaluation}

PPE was performed by experienced medical personnel (a sports cardiologist with support by nurses) either at Stanford University (collegiate athletes), at the athlete's sport club (professional athletes) or in a community setting (precollege athletes), adopting the AHA 14-Element model ${ }^{19}$ (personal/family history and physical examination) with the addition of a 12-lead ECG. Height and weight were measured, and ethnicity was self-reported. The final determination and follow-up of PPE results, including ECGs, were made by an experienced sports cardiologist.

\section{Measurement and classification of blood pressure}

Resting BP was obtained at the time of PPE (cohort 1) or at the echocardiographic examination (cohort 2) using standard protocols. ${ }^{9}{ }^{20}$ Briefly, BP was measured in both arms using an automated oscillometric cuff positioned at the level of the heart (in a majority of observations with Spot Vital Signs, WelAllynlyn, New York, USA), assuring proper cuff-size. Subjects were positioned seated with the forearm rested flat on a supportive surface after at least $5 \mathrm{~min}$ of rest. The higher value in either arm was reported, and in cases of which the initial reading was $\geq 140 / 90 \mathrm{~mm} \mathrm{Hg}$, BP was remeasured in both arms. If still exceeding 140/90 mm Hg after remeasurement, follow-up was recommended with the primary care or sports physician.

BP was categorised per the 2017 US (American Heart Association/American College of Cardiology) and 2018
European (European Society of Cardiology) guidelines for HTN (figure 1A). ${ }^{68}$

\section{Athletic echocardiography screening protocol}

Echocardiography was performed using a commercially available system (iE33; Philips Medical Imaging, Andover, Massachusetts, USA). The echocardiographic protocol included two-dimensional and colour Doppler images in parasternal and apical views. Images were analysed Xcelera workstations. Standard measures of LV size, LV ejection fraction (LVEF) and diastolic parameters were performed. LVM and volume (LVEDV) were calculated using the area-length method in end-diastole from short-axis and apical 4-chamber images and indexed to body surface area (BSA). MVR was derived by dividing LVM and LVEDV. ${ }^{21}$ In diastole, the relative wall thickness (RWT) was calculated from short-axis images as $((2 \times$ average wall thickness $) / \mathrm{LV}$ diameter $)$ and LV sphericity index was determined as (LV length in the apical 4-chamber view/ LV diameter in short-axis view). Mid-wall Lagrangian longitudinal strain (LS, \%) was calculated from apical 4-chamber images by manual tracings of ventricular length $((\mathrm{L} 1-\mathrm{L} 0) / \mathrm{L} 0 \times 100)$, where L1 represents end-systolic and L0 represents end-diastolic wall length. $^{22}$

\section{Statistical analysis}

Continuous data were expressed as mean $\pm S D$, categorical data as number of observations and frequency. For BP, upper 95th percentiles were calculated. Differences between groups were evaluated using one-way analysis of variance or Student's t-test for continuous variables and $\mathrm{X}^{2}$ test for categorical variables. $\mathrm{P}$ values $<0.05$ were considered to be statistically significant. Relationships between BP and descriptive variables were explored using bivariate correlation, univariate linear regression and multiple stepwise linear regression analysis. For regression analyses, unstandardised B-coefficients with 95\% CIs are presented. IBM SPSS Statistics for Windows, V.25.0 (IBM, Armonk, New York, USA) was used for statistical analysis.

\section{RESULTS}

\section{Stanford clinical PPE cohort}

In total, 2733 athletes (table 1) were included after excluding four athletes with previously diagnosed HTN, 95 athletes with
$\mathrm{A}_{\mathrm{s} y+1}$

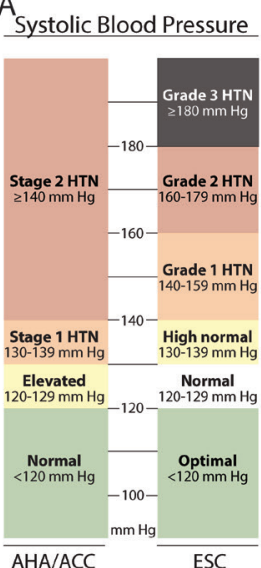

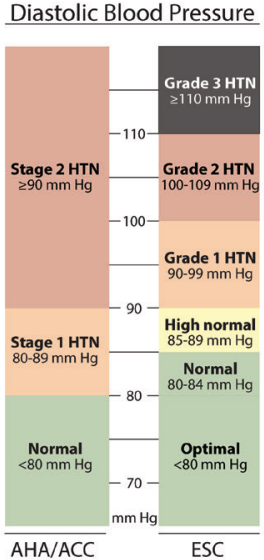

B

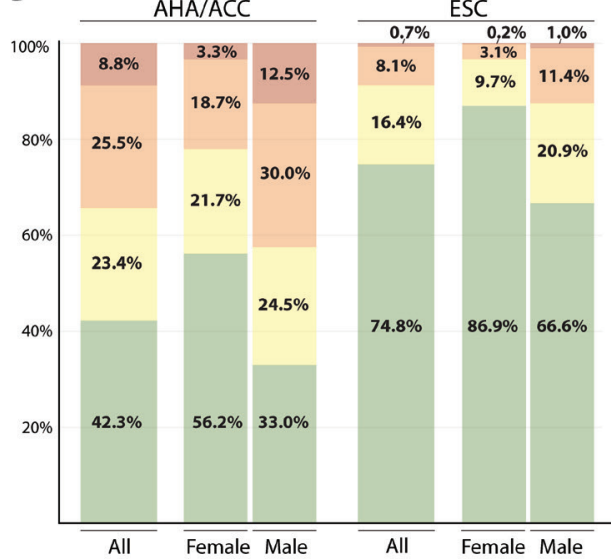

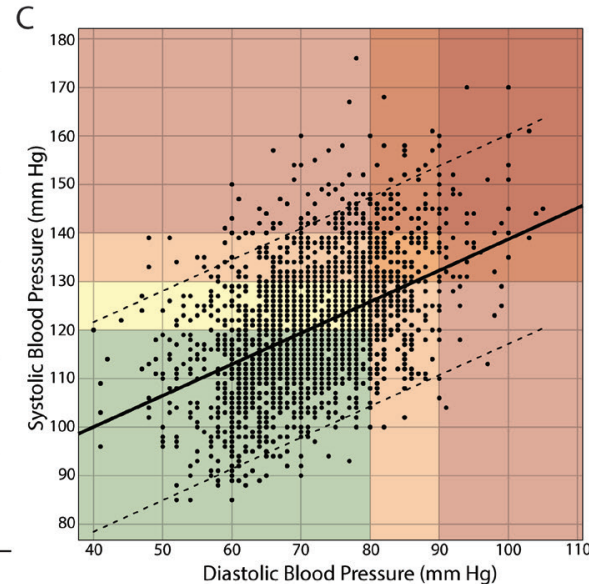

Figure 1 Current thresholds for categorising blood pressure according to US (American Heart Association/American College of Cardiology (AHA/ ACC)) and European (European Society of Cardiology (ESC)) guidelines (panel A) and the proportion of athletes in each blood pressure category according to respective set of guidelines, overall and for each sex (panel B). In panel C, the relationship between systolic and diastolic blood pressure is visualised, where background colour corresponds to the different blood pressure thresholds in the US guidelines. Solid line indicates linear regression line and dashed lines upper and lower limits of the individual $\mathrm{Cl}$. 
Table 1 Characteristics of 2733 athletes presented by seniority level

\begin{tabular}{|c|c|c|c|c|}
\hline & Precollege $(n=953)$ & College $(n=1562)$ & Professional $(n=218)$ & $P$ value* \\
\hline Male $(n, \%)$ & $624(65.5 \%)$ & $807(51.7 \%)$ & $212(97.2 \%)$ & $<0.001$ \\
\hline Age (years) & $15.4 \pm 1.2(13-18)$ & $19.0 \pm 1.1(16-25)$ & $25.7 \pm 3.0(19-35)$ & $<0.001$ \\
\hline Weight (kg) & $64.1 \pm 13.5$ & $73.8 \pm 16.5$ & $106.1 \pm 22.2$ & $<0.001$ \\
\hline Height $(\mathrm{cm})$ & $171.9 \pm 10.2$ & $176.7 \pm 11.8$ & $186.5 \pm 8.5$ & $<0.001$ \\
\hline BMI $\left(\mathrm{kg} / \mathrm{m}^{2}\right)$ & $21.2 \pm 3.6$ & $23.4 \pm 3.4$ & $30.3 \pm 5.0$ & $<0.001$ \\
\hline $\mathrm{BMI} \geq 30(\mathrm{n}, \%)$ & $20(2.1 \%)$ & $67(4.3 \%)$ & $103(47.3 \%)$ & $<0.001$ \\
\hline $\mathrm{BSA}\left(\mathrm{m}^{2}\right)$ & $1.74 \pm 0.21$ & $1.90 \pm 0.25$ & $2.30 \pm 0.26$ & $<0.001$ \\
\hline $\mathrm{SBP}(\mathrm{mm} \mathrm{Hg})$ & $118 \pm 13$ & $122 \pm 13$ & $128 \pm 13$ & $<0.001$ \\
\hline SBP 95th percentile & $140 \mathrm{~mm} \mathrm{Hg}$ & $142 \mathrm{~mm} \mathrm{Hg}$ & $151 \mathrm{~mm} \mathrm{Hg}$ & - \\
\hline $\mathrm{DBP}(\mathrm{mm} \mathrm{Hg})$ & $72 \pm 9$ & $72 \pm 9$ & $74 \pm 9$ & 0.003 \\
\hline DBP 95th percentile & $86 \mathrm{~mm} \mathrm{Hg}$ & $86 \mathrm{~mm} \mathrm{Hg}$ & $89 \mathrm{~mm} \mathrm{Hg}$ & - \\
\hline \multicolumn{5}{|l|}{ Ethnicity $(\mathrm{n}, \%)$} \\
\hline Afro-American & $42(4.4 \%)$ & $153(9.8 \%)$ & $128(58.7 \%)$ & $<0.001$ \\
\hline Asian & $191(20.0 \%)$ & $129(8.3 \%)$ & 0 & \\
\hline Caucasian & $560(58.8 \%)$ & $1127(72.2 \%)$ & $75(34.4 \%)$ & \\
\hline Pacific Islander & $9(0.9 \%)$ & $18(1.2 \%)$ & $5(2.3 \%)$ & \\
\hline Other & $151(15.8 \%)$ & $135(8.6 \%)$ & $10(4.6 \%)$ & \\
\hline
\end{tabular}

${ }^{*} \mathrm{X}^{2}$ or one-way analysis of variance as appropriate. Data presented as mean $\pm \mathrm{SD}$ or number of observations (percentage). For age, range is included.

BMI, body mass index; BSA, body surface area; DBP, diastolic blood pressure; SBP, systolic blood pressure.

initial ECG readings suggesting further follow-up (see online supplementary figure 2), 30 athletes aged $>35$ years and 147 athletes aged $<13$ years. Thirty-five different sports were represented (see online supplementary table 1). Overall, $52.3 \%$ of athletes engaged in either of football (15.1\%), soccer (13.5\%), rowing $(8.8 \%)$, basketball $(7.9 \%)$ or baseball $(7.0 \%)$. Professional athletes differed demographically from others in that $89.0 \%$ were football players, $97.2 \%$ were male and $58.7 \%$ were of African-American decent.

Average SBP was higher in males than in females at all seniority levels, while DBP only differed between sexes in college athletes (figure 2). In unadjusted analysis, average SBP varied from $117 \pm 11$ and $117 \pm 12 \mathrm{~mm} \mathrm{Hg}$ in soccer players and cross-country runners, respectively, to $125 \pm 14 \mathrm{~mm} \mathrm{Hg}$ in football players, which was associated with a corresponding difference in age between sports (figure 3). There was no difference in BP between male football players at a college versus professional level; overall or in subgroups of athletes of either Caucasian or Afro-American ethnicity (see online supplementary table 2).
Of all athletes, 34.3\% $(n=938)$ and $8.8 \%(n=241)$ had an SBP and/or DBP exceeding the HTN thresholds in the US and European guidelines, respectively (figure 1B). With the US guidelines, 6.6 times as many females $(22 \%$ vs $3.3 \%)$ and 3.4 times as many males ( $42 \%$ vs $12.5 \%$ ) exceeded the threshold for HTN compared with using European guidelines. Out of the athletes with a BP exceeding any threshold for HTN by the US guidelines, $38.9 \%$ fulfilled only SBP criteria (ie, DBP $<80 \mathrm{~mm}$ $\mathrm{Hg}$ ), $34.6 \%$ only DBP criteria (ie, SBP $<130 \mathrm{~mm} \mathrm{Hg}$ ) and $26.5 \%$ fulfilled both criteria (figure 1C). Details on the number of athletes by seniority level and sex exceeding respective threshold is presented online supplementary figure 3 .

Male sex, age, height, weight, BMI and Afro-American decent were all weak to moderate, unadjusted correlates of $\mathrm{BP}$, with stronger correlations to SBP than DBP (see online supplementary table 3). When adjusting for sex, age, Afro-American ethnicity, height and BMI, age had no independent association with SBP and BMI was the only factor associated with SBP in all seniority levels (table 2). Male sex was positively and Afro-American
A

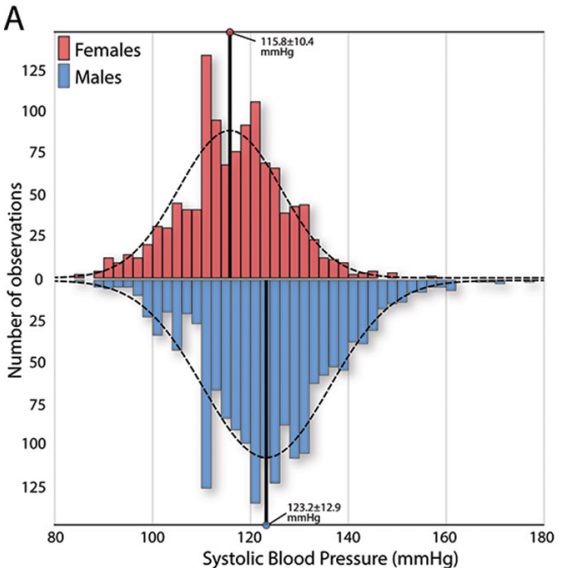

B

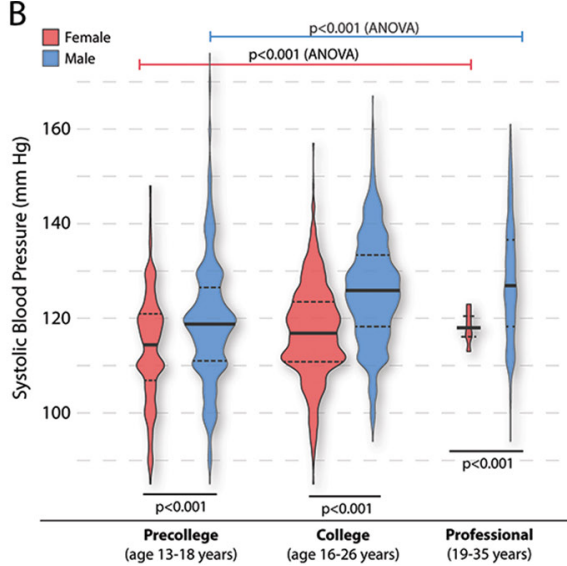

C

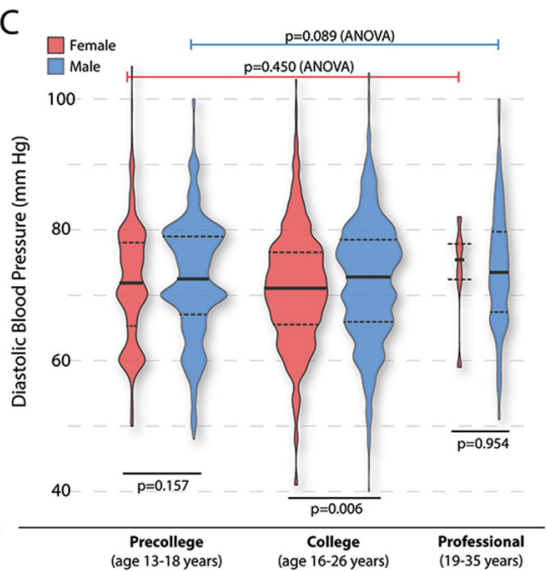

Figure 2 The distribution of systolic blood pressure (SBP) in male (blue) and female (red) athletes with mean values (SD) presented for each sex (panel A). In panels B and C, violin plots of the distribution as well as the 25th, 50th and 75th percentiles for SBP and diastolic blood pressure (DBP) across seniority level for males (blue) and females (red) are presented. P values at the top of each panel for difference between seniority level in females (red) and males (blue), and for difference between sexes at each level at bottom of each panel. 


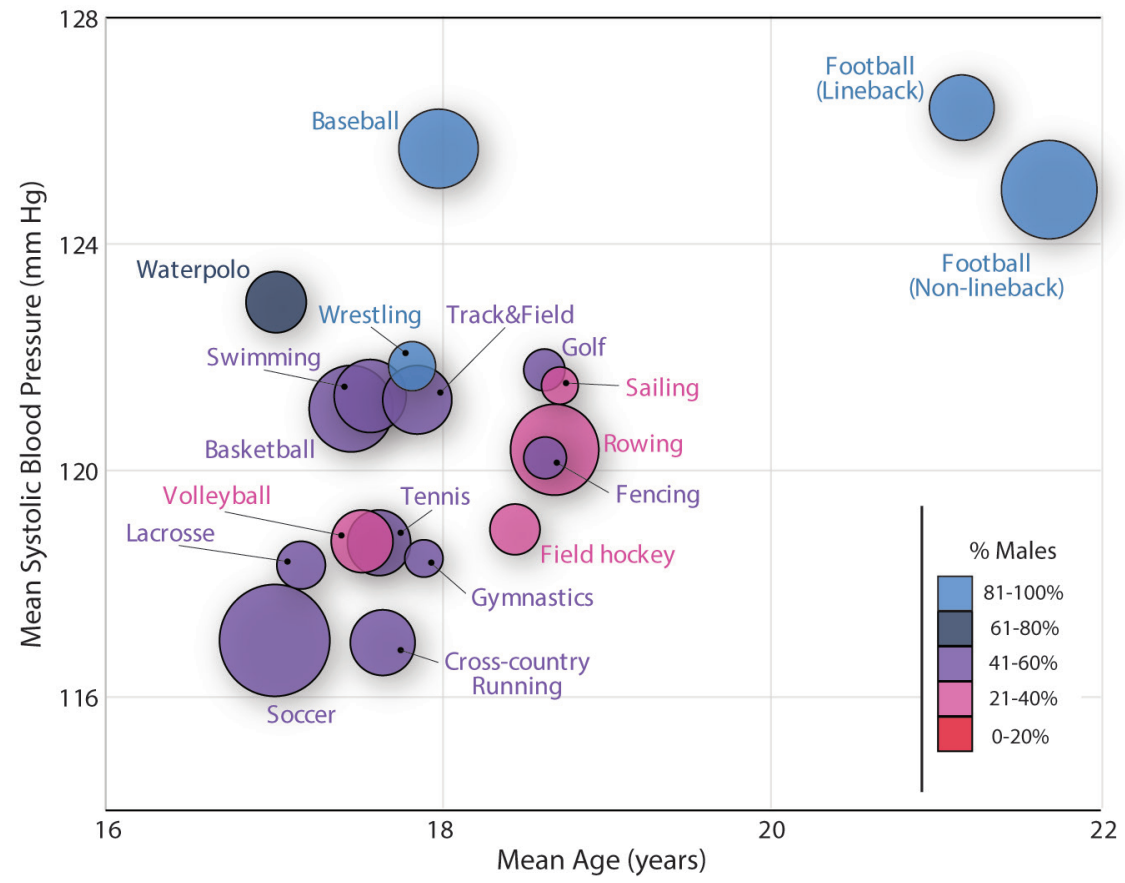

Figure 3 Mean systolic blood pressure (SBP) in all sports with $\geq 40$ athletes, in relation to average age per sport. Circle area is proportional to the number of athletes in each sports category (reference: sailing $n=40$, soccer $n=368$ ) and circle colour indicate the proportion of males in each sport category, as explained in imposed legend.

descent weakly negatively associated with SBP in precollege and college athletes. After excluding professional athletes from the analysis, age was weakly associated with SBP $(B(95 \% \mathrm{CI})=0.47$ $(0.24$ to 0.70$), p<0.001)$ with minimal effect on other associations (see online supplementary table 4 ).

\section{Stanford PPE screening cohort}

In the 304 college athletes (aged 17-26 years) in cohort 2 (table 3), there were 219 football players (all male), 43 basketball players (20 male) and 42 volleyball players (25 male). Overall, 139 (45.7\%) athletes had a BP $\geq 130$ and/or $\geq 80 \mathrm{~mm}$ $\mathrm{Hg}$ and 43 (14.1\%, all male) had a BP $\geq 140$ and/or $\geq 90 \mathrm{~mm}$ $\mathrm{Hg}$. Athletes in higher BP categories had higher BMI, were more likely football players and male and they had larger absolute and indexed LVM. RWT and MVR were greater in higher BP categories (both $\mathrm{p}<0.001$ ), while sphericity index was similar across groups $(p=0.391)$. The difference in MVR between groups is visualised in figure 4. There was no difference in LV function as measured by LVEF and LV LS between groups, while early and late diastolic $(\mathrm{E} / \mathrm{A})$ ratio was lower with increasing $\mathrm{BP}(\mathrm{p}<0.001)$ and there were trends towards lower e' and E-wave velocity in higher BP groups ( $p=0.105$ and 0.102 , respectively).

Bivariate, unadjusted correlations between $\mathrm{BP}$ and LV parameters are presented in online supplementary table 5. In adjusted analyses, multiple stepwise regression (entering sex, age, Afro-American descent, height, BMI, SBP and DBP as independent variables), resulted in a model for MVR $\left(\mathrm{R}^{2} 0.21\right.$, $\mathrm{p}<0.001)$ including Afro-American descent $(\mathrm{B}(95 \% \mathrm{CI})=0.08$ (0.06 to 0.10$), \mathrm{p}<0.001)$, BMI $(\mathrm{B}=0.004$ (95\% CI 0.002 to $0.006), \mathrm{p}=0.001)$ and SBP $(\mathrm{B}=0.002(95 \% \mathrm{CI} 0.001$ to 0.002$)$, $\mathrm{p}=0.001)$. For LVM, the model $\left(\mathrm{R}^{2}=0.56, \mathrm{p}<0.001\right)$ included BMI $(B=3.56$ (95\% CI 2.94 to 4.18), $\mathrm{p}<0.001)$, height $(\mathrm{B}=1.66$ (95\% CI 1.30 to 2.02), $\mathrm{p}<0.001)$, male sex $(\mathrm{B}=20.49$ (95\% CI 11.41 to 29.58$), \mathrm{p}<0.001)$ and Afro-American descent $(\mathrm{B}=7.10$ (95\% CI 0.94 to 13.26$), \mathrm{p}=0.024$ ).

\section{DISCUSSION}

The main finding of our study is that in a large cohort of athletes undergoing PPE, BP exceeded the thresholds recommended in the new US guidelines in one-third of athletes, four times

Table 2 Independent predictors of systolic blood pressure $(\mathrm{mm} \mathrm{Hg})$

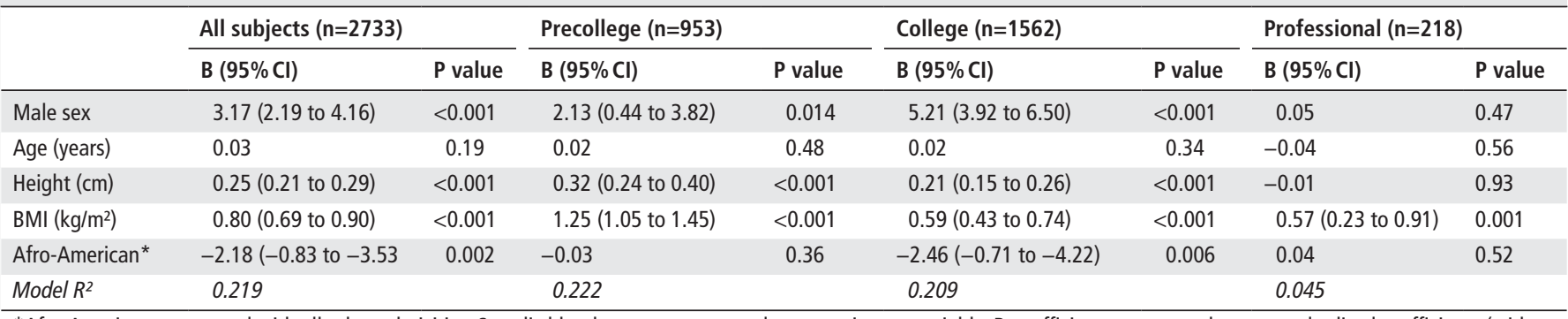

${ }^{*}$ Afro-Americans compared with all other ethnicities. Systolic blood pressure expressed as a continuous variable. B-coefficients correspond to unstandardised coefficients (with $95 \% \mathrm{Cl}$ ) obtained by stepwise multivariable regression analysis. For statistically non-significant correlations, $\mathrm{r}$ and $\mathrm{p}$ values are reported for reference. 
Table 3 Characteristics and echocardiographic measures in 304 collegiate athletes at routine preparticipation echocardiographic screening, presented by blood pressure categories

\begin{tabular}{|c|c|c|c|c|c|}
\hline & $\begin{array}{l}<120 \text { and }<80 \mathrm{~mm} \mathrm{Hg} \\
(n=82)\end{array}$ & $\begin{array}{l}120-129 \text { and }<80 \mathrm{~mm} \mathrm{Hg} \\
(n=83)\end{array}$ & $\begin{array}{l}130-139 \text { and/or } \\
80-89 \mathrm{~mm} \mathrm{Hg}^{*} \\
(\mathrm{n}=96)\end{array}$ & $\begin{array}{l}\geq 140 \text { and } / \text { or } \geq 90 \mathrm{~mm} \mathrm{Hg}^{*} \\
(n=43)\end{array}$ & $P$ value ANOVA \\
\hline Males (n, \%) & $58(70.7 \%)$ & $74(89.2 \%)$ & $89(92.7 \%)$ & $43(100.0 \%)$ & $<0.001$ \\
\hline Age (years) & $18.8 \pm 1.0$ & $18.8 \pm 1.2$ & $19.0 \pm 1.2$ & $19.1 \pm 1.1$ & 0.240 \\
\hline Weight (kg) & $86.0 \pm 16.7$ & $94.1 \pm 16.2$ & $101.6 \pm 19.9$ & $110.7 \pm 21.4$ & $<0.001$ \\
\hline Height $(\mathrm{cm})$ & $186 \pm 9$ & $188 \pm 8$ & $189 \pm 8$ & $191 \pm 7$ & 0.007 \\
\hline $\mathrm{BMI} \geq 30 \mathrm{~kg} / \mathrm{m}^{2}(\%)$ & $6(7.3 \%)$ & $14(16.9 \%)$ & $31(32.3 \%)$ & $20(46.5 \%)$ & $<0.001$ \\
\hline $\mathrm{BSA}\left(\mathrm{m}^{2}\right)$ & $2.10 \pm 0.22$ & $2.20 \pm 0.20$ & $2.28 \pm 0.22$ & $2.38 \pm 0.23$ & $<0.001$ \\
\hline Heart rate (1/min) & $57 \pm 8$ & $57 \pm 8$ & $60 \pm 10$ & $60 \pm 10$ & 0.078 \\
\hline \multicolumn{6}{|l|}{ Ethnicity $(\mathrm{n}, \%) \dagger$} \\
\hline Afro-American & $26(31.7 \%)$ & $20(24.1 \%)$ & $35(36.5 \%)$ & $12(27.9 \%)$ & 0.353 \\
\hline LVMI $\left(g / m^{2}\right)$ & $75.0 \pm 10.2$ & $78.7 \pm 11.7$ & $79.7 \pm 11.4$ & $83.7 \pm 11.4$ & $<0.001$ \\
\hline LVEDV (mL) & $194 \pm 35$ & $206 \pm 40$ & $214 \pm 39$ & $226 \pm 40$ & $<0.001$ \\
\hline LVEDVI (mL/m²) & $92.1 \pm 12.9$ & $93.0 \pm 13.7$ & $93.0 \pm 14.0$ & $93.6 \pm 13.7$ & 0.093 \\
\hline $\operatorname{MVR}(\mathrm{g} / \mathrm{mL})$ & $0.82 \pm 0.09$ & $0.85 \pm 0.10$ & $0.86 \pm 0.10$ & $0.90 \pm 0.10$ & $<0.001$ \\
\hline Sphericity index $\ddagger$ & $1.75 \pm 0.14$ & $1.77 \pm 0.16$ & $1.77 \pm 0.13$ & $1.79 \pm 0.15$ & 0.391 \\
\hline RWT & $0.28 \pm 0.03$ & $0.29 \pm 0.03$ & $0.30 \pm 0.03$ & $0.31 \pm 0.03$ & $<0.001$ \\
\hline LVEF (\%) & $59.3 \pm 3.5$ & $59.0 \pm 5.0$ & $58.3 \pm 4.7$ & $59.2 \pm 4.4$ & 0.514 \\
\hline $\mathrm{LS}_{4 \mathrm{Ch}}(\%)$ & $-19.3 \pm 2.3$ & $-19.3 \pm 2.6$ & $-18.6 \pm 2.2$ & $-18.9 \pm 1.8$ & 0.288 \\
\hline $\mathrm{E}(\mathrm{cm} / \mathrm{s})$ & $83 \pm 16$ & $84 \pm 15$ & $80 \pm 17$ & $77 \pm 16$ & 0.102 \\
\hline$E / A$ & $2.5 \pm 0.7$ & $2.3 \pm 0.6$ & $2.1 \pm 0.5$ & $2.0 \pm 0.5$ & $<0.001$ \\
\hline $\mathrm{e}_{\text {lateral }}^{\prime}$ & $17.3 \pm 3.6$ & $17.6 \pm 3.2$ & $16.9 \pm 3.8$ & $15.9 \pm 3.8$ & 0.105 \\
\hline
\end{tabular}

${ }^{*} \geq 130$ and $/$ or $\geq 90 \mathrm{~mm} \mathrm{Hg}$ corresponds to the threshold defining hypertension in the current US guidelines, while $\geq 140$ and/or $\geq 90 \mathrm{~mm}$ Hg correspond to current European guidelines.

tData on ethnicity were missing for one of the athletes in the $<120 /<80 \mathrm{~mm} \mathrm{Hg}$ group.

¥Sphericity index was determined as (left ventricular (LV) length in the apical 4-chamber view/LV diameter in short-axis view).

ANOVA, analysis of variance; BMI, body mass index; BSA, body surface area; LVM, LVMI and LVEDV, LVEDVI, left ventricular mass and end-diastolic volume with and without indexing for BSA, respectively; MVR, LV mass/volume ratio; RWT, relative wall thickness; LVEF, left ventricular ejection fraction; $\mathrm{LS}_{4 C h^{\prime}}$ longitudinal strain (4-chamber view); E/A, early and late diastolic mitral inflow velocities; $\mathrm{e}^{\prime}{ }_{\text {ateral }}$ early diastolic velocity of the basal lateral left ventricular wall.

as many as with the European guidelines. Although mean BP increased with seniority level, height, BMI and sex rather than age appeared to explain the variability in SBP. Furthermore, we observed that athletes in higher BP categories presented with a greater degree of concentric LV remodelling (greater MVR) and with differences in diastolic function as compared with those in lower BP categories.

\section{$\mathrm{BP}$ in relation to recent HTN guidelines}

There was a large discrepancy in the number of athletes exceeding the thresholds defining HTN using the current US versus European guidelines $(34.3 \%$ vs $8.8 \%)$, especially in females $(22.0 \%$ vs $3.3 \%)$. This reflects the fact that many of the athletes in our cohort had SBP between 130 and $140 \mathrm{~mm} \mathrm{Hg}$ and/or DBP between 80 and $90 \mathrm{~mm} \mathrm{Hg}$ (figure 1C). Current recommendations for BP measurement and HTN management in athletes (published in 2015), ${ }^{9}$ based on the now former US guidelines, ${ }^{20}$ recommend that an initial reading of $\geq 140 / \geq 90 \mathrm{~mm} \mathrm{Hg}(8.8 \%$ in our cohort) should be followed up for HTN. Thus, adopting the thresholds in the new US guidelines for BP screening could have a large impact on the number of athletes recommended for follow-up for HTN.
A clinical diagnosis of HTN is based on BP measurements on at least two separate occasions, ${ }^{6-8}$ which precludes a HTN diagnosis for the athletes in our study. The comprehensive recommendations for BP measurement standardisation within the guidelines ${ }^{68}$ can be challenging to adopt, or fully adhere to in the PPE setting, which may be reflected in the large variability in the protocols used for BP measurement across previous studies reporting BP in athletes. ${ }^{3}$ Although the PPE situation may vary considerably between institutions and countries, common difficulties in acquiring optimal BP measurements include logistic issues (time constraints, many athletes or a team screened at a single occasion) as well as the sometimes stressful, unfamiliar environment for the athlete. By adding ambulatory BP measurements to screening BP in athletes, studies have reported a high prevalence of both white coat $\mathrm{HTN}^{23}$ as well as masked HTN. $^{23} 24$ Thus, a second BP measurement on a separate day (when feasible) or remeasuring BP in all athletes with an initial reading of $\geq 130 / \geq 80 \mathrm{~mm} \mathrm{Hg}$ would probably result in lower reported $\mathrm{BP}$ values. If lower $\mathrm{BP}$ thresholds are to be introduced at PPE, this should come with a high level of adherence to the strict standardisation of BP measurements advocated by the guidelines. ${ }^{689}$ While the findings of the current study need to be 
A

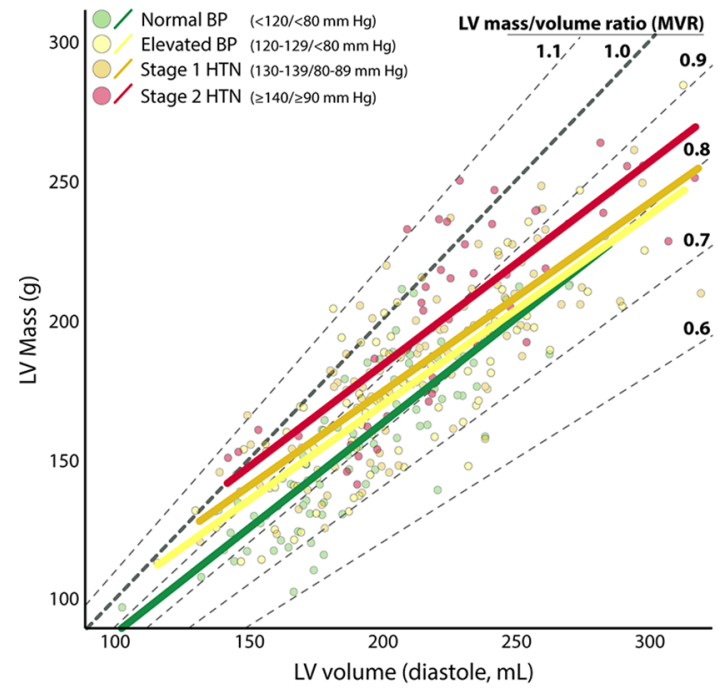

B

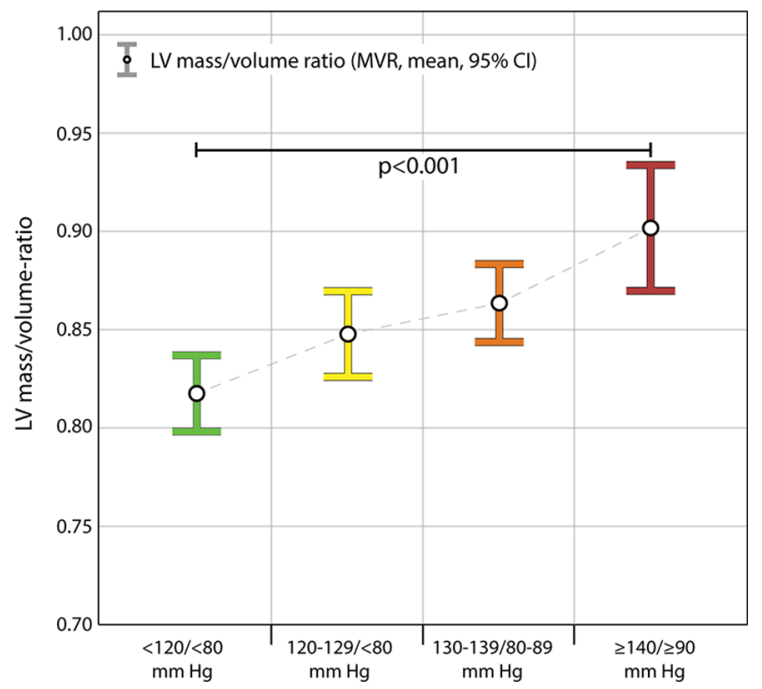

Figure 4 The relation between left ventricular (LV) mass and volume in each athlete (panel A), where dot colour represents what blood pressure category each athlete falls into. The linear relationship between LV mass and volume per blood pressure category is illustrated by coloured lines. The grey diagonal lines correspond to different LV mass/volume ratio (MVR) as noted at end of each line. In panel B, average LV mass/volume ratio (with $95 \% \mathrm{Cls}$ ) in each blood pressure category is displayed.

interpreted in the context of the ongoing challenges in standardisation, our results clearly demonstrate the impact of adapting different thresholds for defining elevated BP or HTN during PPE. While this screening has continuously evolved to more reliably detect underlying cardiac diseases predisposing athletes to sudden cardiac death, ${ }^{19}$ we would like to offer a note of caution before implementing thresholds derived from and evaluated in other settings, without also adopting stricter protocols for measurement. ${ }^{467}$

\section{Factors associated with BP in youth and in young athletes}

SBP increased from precollege to professional athletes, paralleled by an increase in age, height, weight and BMI. Male sex was associated with higher SBP at all seniority levels. While height and BMI were the strongest independent predictors of SBP in our cohort, age was not associated with SBP in adjusted analysis. Our cohort appears representative of contemporary athletes with mean SBP consistent with the averages from 51 original articles on $\mathrm{BP}$ in athletes summarised in a meta-analysis by Berge et $a l^{3}: 114 / 72 \mathrm{~mm} \mathrm{Hg}$ (females) and $121 / 75 \mathrm{~mm} \mathrm{Hg}$ (males), with only marginally higher values in the current study. ${ }^{3}$

The association between height and BP in children and adolescents is established, ${ }^{7}$ as is the higher prevalence of HTN in young subjects with high BMI. ${ }^{1425} 26$ Direct comparison between the prevalence of HTN in our athletes to a general young adult or adolescent US population is difficult, considering the use of different definitions of HTN over time and between studies. ${ }^{725}$ In 761 subjects aged 12-17 years from the National Health and Nutrition Examination Survey, ${ }^{25}$ the prevalence of either high or borderline high BP was 15\%, similar to what has been reported in urban student athletes. ${ }^{26}$ The proportion of athletes exceeding the updated, lower thresholds for HTN in our group of precollege athletes was $36.5 \%$ in males and $25.5 \%$ in females, respectively. These data reinforce a concerning prevalence for elevated BP in the adolescent population and highlights that athletic populations are not immune to cardiovascular risk factors. As both BMI and BP in adolescence and young adulthood have been coupled to adult cardiovascular disease and mortality, ${ }^{4}$
PPE in this population may represent an opportunity for early intervention.

\section{Cardiac remodelling in athletes in relation to BP}

Greater LVM and LVEDV is a common finding in competitive athletes when compared with untrained subjects, ${ }^{15}$ and is considered to represent a physiological remodelling in response to repeated volume load during endurance exercise training. ${ }^{16}$ This remodelling is generally reported as proportional, represented by similar wall/cavity ratio (RWT) $)^{1527}$ and $\mathrm{MVR}^{1728}$ as in untrained subjects. While MVR and RWT in the athletes of the current study were within normal limits compared with previous observations, ${ }^{21}$ athletes with higher BP had higher MVR and RWT than those with lower BP. In addition, SBP assessed during PPE was linearly associated with MVR independently of ethnicity and BMI.

BP level measured in healthy non-athletic children and adolescents has previously been shown to correlate with LV mass ${ }^{13}{ }^{14}$ as well as to LV concentric remodelling, ${ }^{14}$ and recently to predict adult LV concentric hypertrophy. ${ }^{29}$ In addition, Trachsel $e t ~ a l^{24}$ reported higher MVR in middle-aged marathon runners with masked HTN than in normotensive runners. ${ }^{24}$ Our results extend these previous findings to a group of young, presumptuously healthy athletes, suggesting that the process of adverse cardiac remodelling from chronically elevated BP may begin as early as college level. In addition, although LV systolic function at rest was similar across BP categories, those with higher BP presented with lower E/A ratio and trends towards lower E-wave and e' velocities, all markers of diastolic function. Diastolic dysfunction is prevalent in hypertensive heart disease, already at a young age ${ }^{30}$ and is thought to precede more severe LV dysfunction. ${ }^{11}$ Whether our findings reflect subclinical diastolic dysfunction or if they simply are a physiological marker of increased BP remains to be elucidated.

\section{Limitations}

First, the retrospective design holds several limitations. As follow-up was completed by the athlete's primary healthcare team, we were unable to obtain serial BP measurements and review outcomes in 
this cohort. Optimally, BP should have been remeasured at the level of $130 / 80 \mathrm{~mm} \mathrm{Hg}$ in concordance with the current guidelines. Future study designs should endeavour to include ambulatory or in office measurement to validate our findings. Second, echocardiography findings presented from cohort 2 were from a relatively small cohort $(n=304)$, of which a large proportion were male football players, and will require further validation. Third, ethnicity was self-reported with minimal or no guidance, which is inferior to using guided interviewing or, optimally, genetically determined ancestry. Finally, the use of cardiac MRI would provide a more precise measure of LV remodelling; however, this modality is impractical in PPE and echocardiographic-determined measures can be more readily used in the clinical sports cardiological setting.

\section{CONCLUSIONS}

Height, BMI and sex (and not age) explained most of the variability in SBP in our large, heterogenous cohort of athletes, in which a substantial proportion of athletes presented with a BP above the threshold for HTN per current US guidelines. The independent associations between BP and cardiac remodelling suggest that elevated BP in athletes during PPE may signify a clinically relevant condition. However, before considering the lower BP thresholds, efforts for standardising BP measurements at PPE need to be undertaken.

\section{Key questions}

\section{What is already known on this subject?}

- Physical activity is beneficial for primary prevention of hypertension, although smaller previous reports have described relatively high blood pressure in competitive athletes of varying age and sport disciplines.

\section{What might this study add?}

- This study shows that when applying the recently updated US hypertension thresholds, a third of athletes exceeded these thresholds.

- By including athletes over a large age- and body habitus span, we provide insight into the effects of these characteristics on blood pressure in athletes.

- In addition, this study shows that blood pressure level already at a young age may have an impact on cardiac morphology and function in athletes.

\section{How might this impact on clinical practice?}

- Implementing the new US hypertension thresholds at preparticipation evaluation could likely increase the number of athletes for clinical follow-up for hypertension.

- This is important to acknowledge when current guidelines for blood pressure measurements and recommendations in athletic populations are revised.

- High blood pressure at preparticipation evaluation may, however, represent a clinically relevant phenomenon, and careful considerations are warranted in choosing the appropriate blood pressure level where follow-up is warranted.

Acknowledgements The authors would like to thank the personnel and volunteers at Stanford Clinical and Echocardiographic Pre-Participation Evaluation programme for their great efforts.

Contributors $\mathrm{KH}$ and $\mathrm{KJM}$ contributed equally to this study as first authors, and VF and FH contributed equally as senior authors. FH, KH, KJM and VF conceptually planned the study, with valuable additions made by JWC, EA, MA and SPB. EA and
VF built the database. KJM and FH performed the echocardiography, and SPB, KJM $\mathrm{KH}$ and $\mathrm{FH}$ read and/or extracted the data from digital images to a spreadsheet. VF read all ECGs. KH performed the statistical analyses and drafted the first version of the manuscript. All authors contributed in intellectually reviewing and improving the manuscript and approved the final submitted version. All authors agree to be accountable for all aspects of the work.

Funding General funding from the Division of Cardiovascular Medicine at Stanford Cardiovascular Institute was used to finalise this project. KH received funding from the Swedish Society of Medicine and the Fulbright Commission.

Competing interests None declared.

Patient consent for publication Not required.

Ethics approval Stanford Institutional Review Board (IRB no. 25673 and IRB no. 12245).

Provenance and peer review Not commissioned; externally peer reviewed.

\section{REFERENCES}

1 Börjesson M, Onerup A, Lundqvist S, et al. Physical activity and exercise lower blood pressure in individuals with hypertension: narrative review of $27 \mathrm{RCTs}$. $\mathrm{Br}$ J Sports Med 2016;50:356-61.

2 Whelton SP, Chin A, Xin X, et al. Effect of aerobic exercise on blood pressure: a metaanalysis of randomized, controlled trials. Ann Intern Med 2002;136:493-503.

3 Berge HM, Isern CB, Berge E. Blood pressure and hypertension in athletes: a systematic review. Br J Sports Med 2015;49:716-23.

4 Yano Y, Reis JP, Colangelo LA, et al. Association of Blood Pressure Classification in Young Adults Using the 2017 American College of Cardiology/American Heart Association Blood Pressure Guideline With Cardiovascular Events Later in Life. JAMA 2018;320:1774-82.

5 Sundström J, Neovius M, Tynelius P, et al. Association of blood pressure in late adolescence with subsequent mortality: cohort study of Swedish male conscripts. BMJ 2011;342:d643.

6 Whelton PK, Carey RM, Aronow WS, et al. ACC/AHA/AAPA/ABC/ACPM/AGS/APhA/ ASH/ASPC/NMA/PCNA Guideline for the Prevention, Detection, Evaluation, and Management of High Blood Pressure in Adults: Executive Summary: A Report of the American College of Cardiology/American Heart Association Task Force on Clinical Practice Guidelines. J Am Soc Hypertens 2017;2018:579 e1-e73.

7 Flynn JT, Kaelber DC, Baker-Smith CM, et al. Clinical Practice Guideline for Screening and Management of High Blood Pressure in Children and Adolescents. Pediatrics. 2017;140(3):e20171904. Pediatrics 2018:142

8 Williams B, Mancia G, Spiering W, et al. ESC/ESH Guidelines for the management of arterial hypertension. Eur Heart J 2018;2018:3021-104.

9 Black HR, Sica D, Ferdinand K, et al. Eligibility and Disqualification Recommendations for Competitive Athletes With Cardiovascular Abnormalities: Task Force 6: Hypertension: A Scientific Statement from the American Heart Association and the American College of Cardiology. Circulation 2015;132:e298-302.

10 Messerli FH, Rimoldi SF, Bangalore S. Changing definition of hypertension in guidelines: how innocent a number game? Eur Heart J 2018;39:2241-2.

11 Drazner MH. The progression of hypertensive heart disease. Circulation 2011;123:327-34

12 Post WS, Larson MG, Levy D. Impact of left ventricular structure on the incidence of hypertension. The Framingham Heart Study. Circulation 1994;90:179-85.

13 Daimee UA, Lande MB, Tang W, et al. Blood pressure and left ventricular mass index in healthy adolescents. Blood Press Monit 2017;22:48-50.

14 Pieruzzi F, Antolini L, Salerno FR, et al. The role of blood pressure, body weight and fat distribution on left ventricular mass, diastolic function and cardiac geometry in children. J Hypertens 2015;33:1182-92.

15 Utomi V, Oxborough D, Whyte GP, et al. Systematic review and meta-analysis of training mode, imaging modality and body size influences on the morphology and function of the male athlete's heart. Heart 2013;99:1727-33.

16 Arbab-Zadeh A, Perhonen M, Howden E, et al. Cardiac remodeling in response to 1 year of intensive endurance training. Circulation 2014;130:2152-61.

17 Luijkx T, Cramer MJ, Buckens CF, et al. Unravelling the grey zone: cardiac MRI volume to wall mass ratio to differentiate hypertrophic cardiomyopathy and the athlete's heart. Br J Sports Med 2015;49:1404-9.

18 Petersen SE, Selvanayagam JB, Francis JM, et al. Differentiation of athlete's heart from pathological forms of cardiac hypertrophy by means of geometric indices derived from cardiovascular magnetic resonance. J Cardiovasc Magn Reson 2005;7:551-8.

19 Maron BJ, Friedman RA, Kligfield P, et al. Assessment of the 12-lead electrocardiogram as a screening test for detection of cardiovascular disease in healthy general populations of young people (12-25 years of age): a scientific statement from the American Heart Association and the American College of Cardiology. J Am Coll Cardiol 2014;64:1479-514

20 Chobanian AV, Bakris GL, Black HR, et al. The Seventh Report of the Joint National Committee on Prevention, Detection, Evaluation, and Treatment of High Blood Pressure: the JNC 7 report. JAMA 2003;289:2560-72. 


\section{Special populations}

21 Byrd BF, Wahr D, Wang YS, et al. Left ventricular mass and volume/mass ratio determined by two-dimensional echocardiography in normal adults. J Am Coll Cardiol 1985;6:1021-5. 3rd.

22 Kobayashi Y, Ariyama M, Kobayashi Y, et al. Comparison of left ventricular manual versus automated derived longitudinal strain: implications for clinical practice and research. Int J Cardiovasc Imaging 2016;32:429-37.

23 Berge HM, Andersen TE, Solberg EE, et al. High ambulatory blood pressure in male professional football players. Br J Sports Med 2013;47:521-5.

24 Trachsel LD, Carlen F, Brugger N, et al. Masked hypertension and cardiac remodeling in middle-aged endurance athletes. J Hypertens 2015;33:1276-83.

25 Kit BK, Kuklina E, Carroll MD, et al. Prevalence of and trends in dyslipidemia and blood pressure among US children and adolescents, 1999-2012. JAMA Pediatr 2015;169:272-9.
26 Kropa J, Close J, Shipon D, et al. High Prevalence of Obesity and High Blood Pressure in Urban Student-Athletes. J Pediatr 2016;178:194-9.

27 Caselli S, Vaquer Sequì A, Lemme E, et al. Prevalence and Management of Systemic Hypertension in Athletes. Am J Cardiol 2017:119:1616-22.

28 Caselli S, Di Paolo FM, Pisicchio C, et al. Three-dimensional echocardiographic characterization of left ventricular remodeling in Olympic athletes. Am J Cardiol 2011;108:141-7.

29 Zhang T, Li S, Bazzano L, et al. Trajectories of Childhood Blood Pressure and Adult Left Ventricular Hypertrophy: The Bogalusa Heart Study. Hypertension 2018;72:93-101.

30 Palazzuoli A, Gennari L, Calabria P, et al. Left ventricular hypertrophy differences in male professional runners and in young patients suffering from mild hypertension. Blood Press 2004:13:14-19. 